\title{
Aplicações Tecnológicas da Metodologia Cristalográfica
}

\author{
William B. Fernandes, Hamilton B. Napolitano, Caridad Noda- \\ Perez, Felipe T. Martins \& Carlito Lariucci
}

Com o intuito de se conhecer a estrutura molecular e cristalina dos compostos químicos para posteriores análises físico-químicas e estudos de aplicação, lança-se mão da cristalografia como metodologia científica, que permite conhecer a estrutura de qualquer composto químico cristalino sem nenhuma informação a priori. Esta metodologia está baseada em conceitos como difração de raios X por materiais cristalinos, simetria, lei de Bragg e transformada de Fourier. O presente trabalho tem como finalidade apresentar as principais etapas do trabalho cristalográfico, desde a coleta de dados em monocristais, solução e refinamento da estrutura até as etapas de análise e validação do modelo cristalográfico obtido e posterior depósito no banco de dados apropriados.

Palavras-chave: estrutura molecular, difração de raios $X$, interações intermoleculares.

In order to know the molecular and crystalline structure of chemical compounds for further physical-chemical analysis and application studies, launches hand of crystallography as a scientific methodology that allows knowing the structure of any crystalline chemical compound without any a priori information. This methodology is based on concepts such as X-ray diffraction by crystalline materials, symmetry, Bragg's law and Fourier transform. This work aims to present the main steps of crystallographic methodology, since the data collection from a single crystal, solution and refinement of structures until the steps of analysis and validation of crystallographic model, and deposit in appropriate crystallographic database.

Keywords: . molecular structure, $X$-ray diffraction, intermolecular interactions. 


\section{Introdução}

O conhecimento do arranjo dos átomos em uma molécula (estrutura molecular) e da posição relativa de todas as moléculas em um cristal (estrutura cristalina) para substâncias obtidas tanto sinteticamente como isolados de fontes naturais, é extremamente útil para o entendimento das propriedades químicas, físico-químicas e biológicas dos compostos para os mais variados ramos da ciência ${ }^{1,2}$. Na Física e na Ciência dos Materiais, é fundamental a relação entre propriedades físicas e a estrutura interna dos sólidos; em Química as características estéricas de novos compostos ou complexos só podem, em muitos casos, serem conhecidas através da determinação de sua estrutura; em Biologia e Bioquímica a atividade funcional de uma biomolécula está intimamente relacionada com sua estrutura tridimensional; em Medicina e Farmacologia a ação de certos fármacos envolve interações entre fármacoreceptor e a maneira como isto acontece é determinada pela estrutura de ambos componentes ${ }^{1,3,4}$.

Com o interesse em conhecer a matéria a nível atômico, torna se necessário o desenvolvimento de ferramentas que possibilitem a obtenção de informações a este nível de resolução. Entretanto, poucas são as metodologias rotineiramente disponíveis quando se faz necessário saber a distribuição espacial relativa dos átomos constituintes de uma dada molécula. Estas metodologias incluem: Difração de Raios X (DRX) por Monocristais, Difrações de Nêutrons, Ressonância Magnética Nuclear, Espalhamento de Raios X a Baixo Ângulo (SAXS), Microscopia Eletrônica, Modelagem Teórica e outros. Dentre estas, o método cristalográfico apresenta-se como o mais adequado devido à alta resolução com que se pode descrever a densidade eletrônica ${ }^{2,5}$. Este método está baseado no fenômeno da difração, e fornece o conhecimento da disposição tridimensional dos átomos na estrutura molecular para compostos no estado cristalino quando expostos a radiação $\mathrm{X}$, não necessitando de nenhum conhecimento a respeito do composto a priori ${ }^{3,4}$.

Os dados obtidos através de experimentos de difração de raios $\mathrm{X}$ consistem apenas nas intensidades e as posições dos feixes difratados, a partir dos quais se pode obter a amplitude dos fatores de estrutura $|\mathrm{F}(\mathrm{h})|$. Toda informação explícita das fases dos fatores de estrutura, $\varphi(\mathbf{h})$, é perdida durante o experimento, o que impossibilita a determinação analítica da estrutura a partir das intensidades medidas. As posições atômicas são determinadas pelos valores máximos da função de densidade eletrônica $\rho$ (r) (Eq. (1) $)^{3,6}$, onde $V$ é o volume da cela unitária e $\mathrm{F}(\mathbf{h})$ é o fator de estrutura na forma complexa, sendo seu módulo $|\mathrm{F}(\mathbf{h})|$ proporcional à intensidade da reflexão medida para direção $h k l$. A quantidade h.r corresponde ao produto escalar entre o vetor espalhamento no espaço recíproco $\mathbf{h}$ e o vetor posição no espaço direto $\mathbf{r}$.

$$
\rho(\mathbf{r})=\frac{1}{V} \sum_{\mathbf{h}} F(\mathbf{h}) \exp [-2 \pi i(\mathbf{h} . \mathbf{r})]=\frac{1}{V} \sum_{\mathbf{h}}|F(\mathbf{h})| \exp [-2 \pi i(\mathbf{h} . \mathbf{r})+i \varphi(\mathbf{h})]
$$

Para que essa função seja conhecida, torna-se necessário o conhecimento dos fatores de estrutura $F(\mathbf{h})$ (amplitude e fase) de cada reflexão coletada. Nesse sentido, o trabalho de caracterização estrutural da amostra cristalina é a construção da densidade eletrônica a partir das amplitudes e fases dos fatores de estrutura. Uma vez que estas informações são obtidas, e as posições dos átomos precisamente conhecidas, são calculadas as distâncias interatômicas, os ângulos de ligação, ângulos conformacionais, e as características moleculares de interesse, como por exemplo, a planaridade, estabilidade de um grupo particular de átomos e informações estéreoquímicas e estruturais desejadas ${ }^{7,8}$. São calculadas ainda as interações intermoleculares envolvidas no empacotamento molecular do cristal, sendo este, um ramo da ciência conhecido como Química Supramolecular . Frequentemente surgem problemas durante o refinamento de uma estrutura, tais como coleta de cristais geminados (twinning) e problemas de desordem cristalográfica, e alguns procedimentos e avaliações no sentido de modelar e resolver estes problemas necessitam ser executados ${ }^{10}$. Desta forma, o trabalho de elucidação estrutural por cristalografia de raios $\mathrm{X}$ em monocristais, é um trabalho interativo e multidisciplinar, e envolve as seguintes etapas: coleta, processamento e redução dos dados; solução e refinamento da estrutura; validação e análise do modelo cristalográfico; depósito no banco de dados apropriados ${ }^{2,3}$.

\section{A Difração de Raios X por Cristais}

A difração pode ser definida como o fenômeno de 
perturbação do deslocamento de uma onda devido à interação com um obstáculo. Mais especificamente, a DRX pode ser entendida como a alteração da direção de propagação, amplitude e fase da radiação eletromagnética cujo comprimento de onda $(\lambda)$ varia entre $0,1 \AA$ e $100 \AA$ como consequência da interação com centros espalhadores eletrônicos periodicamente arranjados em um cristal. Para que haja DRX por um cristal, raios $X$ espalhados devem estar em fase para interferirem construtivamente e terem suas amplitudes resultantes regidas pelo princípio de superposição de ondas. Para estarem em fase, a diferença de percurso entre os feixes difratados por centros espalhadores deve equivaler a um número inteiro $n$ de comprimentos de onda ( $n \lambda)$. O termo $n$ representa a ordem de difração, o qual deve ser obrigatoriamente um número inteiro. A construção geométrica que relaciona esta observação com o ângulo de difração $\theta$ e a distância $d$ entre os centros espalhadores periodicamente arranjados é conhecida como Lei de Bragg e é dada por:

$$
2 d \operatorname{sen} \theta=n \lambda .
$$

Uma vez satisfeita esta condição, o que necessariamente implica que os centros espalhadores e a radiação espalhada possuem grandezas espaciais de magnitude similar, ondas refletidas a partir de centros espalhadores periodicamente ordenados estarão todas em fase e resultarão em feixes difratados. A função matemática que descreverá tais feixes difratados dependerá da natureza e estrutura dos centros espalhadores. Portanto, quando difratados, raios $\mathrm{X}$ informam quais e como os átomos espalhadores estão periodicamente arranjados no espaço, o que permite a determinação da estrutura intramolecular e intermolecular, e também as dimensões da cela unitária cristalográfica.

Quando há uma relação de fase definida entre o vetor do campo elétrico incidente $E_{\text {in }}$ de uma radiação eletromagnética e a aceleração causada em um elétron devido à interação com esta radiação, qualquer elétron de carga $e$ e massa $m$ é acelerado com uma aceleração $a$ :

$$
a=\frac{e}{m} E_{\text {in. }}
$$

Por sua vez, o elétron acelerado atuará como uma fonte emissora de radiação sob desaceleração. Este é o princípio do espalhamento Thomson, em que uma onda interage com uma partícula e é espalhada com a mesma frequência e comprimento de onda da radiação incidente. O espalhamento Thomson é referenciado também como espalhamento coerente e é aquele observado no fenômeno de DRX por cristais, embora o espalhamento Compton incoerente também ocorra como parte da radiação espalhada de fundo. Entretanto, o espalhamento Compton, caracterizado pela colisão entre duas partículas (fóton e elétron) com alteração na frequência e comprimento de onda da radiação espalhada, não resulta no padrão discreto de DRX, pois não há uma relação de fase bem definida neste evento de interação partícula-partícula.

A radiação espalhada será caracterizada por um vetor de campo elétrico $E_{\text {esp }}$ que poderá se orientar em qualquer direção no plano normal à direção de propagação da radiação eletromagnética. Esta aleatoriedade da direção de $E_{\text {esp }}$ se deve ao fato do feixe incidente também não ser polarizado. Considerando que a polarização parcial $p$ de $E_{\text {esp }}$ é uma função do ângulo de difração $\theta, \varepsilon_{0}$ é uma constante eletromagnética fundamental da ordem de $8,854.10^{-12} \mathrm{~F} \mathrm{~m}^{-1}$, $c$ é a velocidade de propagação da radiação eletromagnética e $r$ é a distância entre o elétron e o ponto de detecção da radiação espalhada, $E_{\text {esp }}$ será dado por:

$$
E_{\text {esp }}=\frac{e^{2}}{4 \pi \varepsilon_{0} r m c^{2}} \frac{\left[1+\cos ^{2}(2 \theta)\right]}{2} E_{\text {in. }} .
$$

Se definirmos a habilidade de um elétron em espalhar a radiação eletromagnética como o fator de espalhamento eletrônico $f_{\mathrm{e}}$ :

$$
f_{\mathrm{e}}=\frac{e^{2}}{4 \pi \varepsilon_{0} r m c^{2}},
$$

temos uma nova expressão para a amplitude da radiação espalhada $E_{\text {esp }}$ :

$$
E_{\text {esp }}=f_{\mathrm{e}} \frac{\left[1+\cos ^{2}(2 \theta)\right]}{2} E_{\text {in }} .
$$

Correções devidas à polarização parcial do vetor de campo elétrico da radiação espalhada devem ser realizadas, principalmente quando cristais monocromadores são utilizados para seleção de determinado comprimento 
de onda dos raios $\mathrm{X}$. A polarização parcial induzida pelo cristal monocromador afetará o espalhamento subsequente pelo cristal em estudo, comprometendo a magnitude da radiação espalhada. Neste caso, o termo referente à polarização $p$ deve ser reescrito como:

$$
p=\frac{\left[1+K \cos ^{2}(2 \theta)\right]}{1+K}
$$

onde Kérazão entre os feixes paralelo e normal à superfície do plano espalhador da radiação na fonte monocromática. Estes dois feixes são derivados da decomposição do vetor de campo elétrico em dois componentes, um paralelo à superfície do plano espalhador, dependente apenas da densidade eletrônica no plano (feixe $\pi$ ou $I_{\|}$), e outro perpendicular a esta superfície (feixe $\sigma$ ou $I_{\perp}$ ), o qual depende tanto da densidade eletrônica no plano quanto do ângulo de difração $\theta$. Este último componente é responsável pelo decréscimo da radiação espalhada devido ao fator de polarização. Sua completa anulação e, consequentemente, a anulação de metade da radiação espalhada decomposta nos dois componentes $I_{\|}$e $I_{\perp}$ de mesma magnitude ocorre quando $2 \theta=90^{\circ}$. Considerando o ângulo de difração $\theta_{\mathrm{m}}$ do cristal monocromador, uma aproximação muito utilizada para o valor de $K$ é:

$$
K=\cos \left(2 \theta_{\mathrm{m}}\right)
$$

Uma vez aplicada correção devida à polarização parcial da radiação espalhada, podemos expressar a radiação espalhada $E_{\text {esp }}$ por um elétron como:

$$
E_{\text {esp }}=f_{\mathrm{e}} E_{\text {in }} .
$$

Para uma distribuição discreta de $n$ elétrons, a radiação espalhada $E_{\text {esp }}$ total relativa à radiação incidente $E_{\text {in }}$ será dada por:

$$
\frac{E_{\text {esp }}}{E_{\text {in }}}=\sum_{n} f_{\mathrm{e}} e^{i \phi_{n}},
$$

onde o termo exponencial se refere à relação de fase definida como o número ou frações de comprimentos de onda que separam os elétrons discretamente distribuídos no espaço.

Enquanto a habilidade de um elétron em espalhar a radiação eletromagnética é definida como $f_{\mathrm{e}}$, a habilidade de uma distribuição eletrônica contínua, como aquela de átomos reais, em espalhar a radiação eletromagnética pode ser entendida como:

$$
f_{\mathrm{e}} \rho(\mathbf{r}) d(\mathbf{r})
$$

onde o produto $\rho(\mathbf{r}) d(\mathbf{r})$ é o número médio de elétrons confinados no elemento de volume $d(\mathbf{r})$. Portanto, $\rho(\mathbf{r})$ pode ser referenciada como a função de densidade eletrônica de uma distribuição eletrônica.

Agora, reescrevendo a equação da radiação espalhada $E_{\text {esp }}$ total relativa à radiação incidente $E_{\text {in }}$ para uma distribuição contínua de elétrons em que a habilidade de um centro espalhador em espalhar a radiação será dada pela equação anterior, e expressando a fase relativa no termo exponencial em função do vetor de espalhamento $\Delta \mathbf{k}$ e do centro espalhador $\mathbf{r}$ como $e^{i \phi n}=e^{i \Delta \mathbf{k} \mathbf{r}}$, temos:

$$
\frac{E_{\text {esp }(\Delta \mathbf{k})}}{E_{\text {in }}}=\int_{\mathbf{r}} f_{\mathrm{e}} \rho(\mathbf{r}) e^{i \Delta \mathbf{k} \mathbf{r}} d(\mathbf{r}) .
$$

Agora, a radiação espalhada é função do vetor de espalhamento $\Delta \mathbf{k}$, cuja direção de espalhamento é dada pelos índices $h k l$. Uma vez que a integração não é sobre a variável $r$ embutida em $f_{\mathrm{e}}$, nós podemos extrair este termo da integral. Em adição, a expressão

$$
\frac{E_{\mathrm{esp}(\Delta \mathbf{k})}}{E_{\text {in }} f_{\mathrm{e}}}
$$

representa a habilidade relativa dos centros espalhadores de uma cela unitária em espalhar radiação eletromagnética quando comparada com a habilidade de um único elétron. Esta expressão especifica a amplitude da difração em uma dada direção de espalhamento indexada por hkl. Então, podemos definir a expressão anterior como o fator de estrutura $F_{h k l}$ de um máximo de difração correspondente ao ponto da rede recíproca definido pelos integradores $h k l$.

Através da substituição da representação vetorial $\mathbf{r}$ dos centros espalhadores no termo exponencial por coordenadas fracionárias xyz e vetores cristalográficos $a b c$, representando o ponto r como:

$$
x \mathrm{a}+y \mathrm{~b}+z \mathrm{c}
$$

e o volume $d \mathbf{r}$ em função do volume $V$ da cela unitária como: 
$V d x d y d z$

e, por fim, assumindo a proporcionalidade entre o vetor de espalhamento $\Delta \mathbf{k}$ e o vetor de rede recíproca $G_{h k l}$ como sendo:

$$
\Delta \mathbf{k}=2 \mathrm{p} \mathrm{G}_{h k l},
$$

onde $G_{h k l}$ pode ser definido em termos de vetores cristalográficos da rede recíproca $\mathrm{a}^{*} \mathrm{~b}^{*} \mathrm{c}^{*}$ como:

$$
\mathrm{G}_{h k l}=d_{\mathrm{hkl}}^{-1}=h \mathrm{a}^{*}+k \mathrm{~b}^{*}+l \mathrm{c}^{*},
$$

temos o fator de estrutura $F_{h k l}$ como:

$$
F_{h k l}=V \int_{x=0}^{x=1} \int_{y=0}^{y=1} \int_{z=0}^{z=1} \rho(x y z) e^{2 \pi i(h x+k y+l z)} d x d y d z .
$$

A integração sobre a função de densidade eletrônica através das coordenadas da cela unitária deve ser realizada sobre todo o conteúdo da mesma, o que justifica os limites da integração indo de zero a um. Desta forma, a função de densidade eletrônica, outrora representada na forma vetorial por $\rho(\mathbf{r})$, é também expressa agora como função das coordenadas fracionárias xyz.

Fatores de estrutura $F_{h k l}$ podem ser expressos em função de fatores de espalhamento atômico. Considerando que um elétron isolado espalha raios $\mathrm{X}$ com uma determinada intensidade definida pelo fator de espalhamento Thomson, um átomo contendo $\mathrm{Z}$ elétrons espalharia raios $\mathrm{X}$ com intensidade igual ao produto entre $\mathrm{Z}$ e a intensidade de um único elétron isolado, conforme descrito anteriormente. Entretanto, devido às distâncias entre os átomos serem da ordem de grandeza do comprimento de onda dos raios $\mathrm{X}$, as ondas que os elétrons espalham interferem umas com as outras, de forma que só haverá uma dada intensidade correspondendo a todos os elétrons de um átomo na direção de incidência dos raios X. Para o espalhamento em outras direções observa-se interferência parcialmente destrutiva devido ao distanciamento entre os elétrons de um átomo culminar em diferenças de percurso da radiação espalhada. Assim, a amplitude total do feixe espalhado por um átomo, a qual recebe contribuições de todos os seus elétrons, ou seja, é proporcional ao número atômico e dependente dos espaçamentos intereletrônicos, decresce com o aumento do ângulo de espalhamento. Esta habilidade de um átomo em espalhar os raios $\mathrm{X}$ é definida como o fator de espalhamento atômico $f_{j}$ e constitui a amplitude espalhada pela distribuição de elétrons de um átomo normalizada em relação à amplitude espalhada por um elétron isolado, sob condições idênticas. Fatores de espalhamento atômico podem assumir como valor máximo Z (número atômico do átomo) na ocasião dos elétrons espalharem a radiação em fase, na direção de incidência do feixe de raios $\mathrm{X}(2 \theta=0)$. Para os feixes espalhados na direção da incidência, os raios X estarão em fase e a amplitude resultante deriva do principio de sobreposição de ondas. Quando o ângulo de difração é diferente de zero, as trajetórias dos raios espalhados são diferentes e a diferença de fase resulta em interferência. Considerando uma molécula, a onda total espalhada por todos os átomos é dada pela soma vetorial das contribuições individuais de cada átomo.

Em prática, o fator de espalhamento atômico significa a tendência de um átomo para espalhar a radiação $X$, a um dado ângulo e certo $\lambda$. Assim, valores tabelados são encontrados para razões de $\operatorname{sen} \theta / \lambda$, expressos como o quociente entre a amplitude da onda espalhada por um átomo sobre a amplitude da onda espalhada por um elétron. De maneira similar ao fator de espalhamento atômico, o fator de estrutura é um quociente de duas amplitudes: a amplitude da onda espalhada por todos os átomos da cela unitária e a amplitude da onda espalhada por um elétron isolado, onde a magnitude deste fator depende unicamente da posição relativa de todos os átomos na cela unitária e de seus respectivos fatores de espalhamento. Então, é conveniente expressar os fatores de estrutura $F_{h k l}$ em função dos valores de $f_{j}$ de cada átomo, o qual embute a função de densidade eletrônica do átomo em um certo volume determinado pelo seu raio atômico. Então, a equação anterior é reescrita como:

$$
F_{h k l}=\sum_{j} f_{j} e^{2 \pi i(h x j+k y j+l z j)},
$$

onde o somatório deve ser efetuado sobre todos os átomos da cela unitária. Uma vez que os átomos da cela unitária podem estar relacionados por elementos de simetria do grupo espacial, a equação anterior pode ser simplificada. Os fatores de espalhamento atômico devem também ser 
corrigidos em função da temperatura. Em um modelo isotrópico de deslocamento, em que as vibrações térmicas são esfericamente simétricas em todas as direções, o fator de temperatura $\mathrm{T}_{\mathrm{j}, \theta}$ de um átomo para qualquer conjunto de planos de rede $(h k l)$ dependerá do espaçamento interplanar e da magnitude de vibração perpendicular aos planos. Então, o fator de espalhamento atômico corrigido $\left(f_{j}\right)_{\mathrm{T}}$ em função da temperatura será dado por:

$$
\left(f_{j}\right)_{\mathrm{T}}=f_{j} \mathrm{~T}_{\mathrm{j}, \theta}
$$

onde $\mathrm{T}_{\mathrm{j}, \theta}$ é dado por:

$$
\mathrm{T}_{\mathrm{j}, \theta}=e^{-B_{j}\left(\operatorname{sen}^{2} \theta / \lambda^{2}\right)},
$$

e $B_{j}$ assume a grandeza:

$$
8 \mathrm{p}^{2}<u^{2}>
$$

onde $<u^{2}>$ representa o deslocamento quadrático médio do átomo $j$.

Em cristais moleculares, os átomos estão ligados covalentemente uns aos outros e, portanto, forças atrativas e repulsivas sobre ligações químicas distorcem a distribuição da densidade eletrônica de tal forma que um modelo isotrópico de deslocamento atômico deve ser aperfeiçoado para um modelo anisotrópico de vibração térmica. Neste modelo, os átomos têm diferentes liberdades de deslocamento em três direções ortogonais (mutuamente perpendiculares). Então, neste modelo mais realístico de deslocamento térmico, um único parâmetro térmico isotrópico $B_{j}$ é substituído por seis parâmetros térmicos anisotrópicos $U_{i j}$, três deles definindo a forma da representação elipsoidal do átomo $j$ e três especificando sua orientação. Neste caso, a expressão para o fator de temperatura anisotrópico $\mathrm{T}_{\mathrm{j}, \theta}$ será dada por:

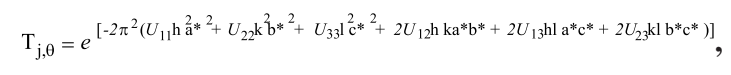

A função de densidade eletrônica $\rho(x y z)$ varia periodicamente de uma cela unitária a outra através do cristal, como consequência do ordenamento periódico dos átomos no estado cristalino. Devido a esta periodicidade, $\rho(x y z)$ pode ser descrita por uma série de Fourier tridimensional. Portanto, o padrão de DRX é a transformada de Fourier $\boldsymbol{T}$ da função de densidade eletrônica. Pelo teorema de Fourier, a função de densidade eletrônica será a transformada inversa $\boldsymbol{T}^{\mathbf{1}}$ do padrão de DRX, como exemplificado abaixo:

$$
\begin{aligned}
& F_{h k l}=T \rho(x y z), \\
& \rho(x y z)=T^{-1} F_{h k l} .
\end{aligned}
$$

A partir do padrão de DRX, composto pela amplitude e fase intrínseca dos fatores de estrutura $F_{h k l}$ difratados em direções de espalhamento discretas e definidas, a função de densidade eletrônica pode ser então calculada para cada ponto da cela unitária através de suas coordenadas fracionárias por meio da síntese de Fourier dada pela relação:

$$
\rho(x y z)=V^{-1} \sum_{h=\infty}^{h=+\infty k=+\infty l=+\infty} \sum \sum_{k=-\infty} F_{h k l} e^{-2 \pi i(h x+k y+l z)} .
$$

Como mencionado anteriormente, o padrão de DRX é discreto e não contínuo, sendo caracterizado por fatores de estrutura $F_{h k l}$ em posições indexadas. Por esta razão, a integral tripla se degenera dentro de somatórios na equação anterior sobre todos os valores possíveis de índices dos vetores de espalhamento. A intensidade da radiação espalhada é uma função inversamente proporcional ao ângulo de difração $\theta$, sendo que, para um dado comprimento de onda $\lambda$ da radiação incidente, somente os pontos da rede recíproca caracterizados pelo vetor de rede recíproca $G_{h k l}$ cujas magnitudes satisfazem a relação:

$$
\mathrm{G}_{h k l} \leq 2 \lambda^{-1}
$$

poderão ser detectados em um experimento de DRX. O termo $2 \lambda^{-1}$ é o raio da esfera limite, a qual representa uma construção geométrica originada da rotação do cristal sobre a esfera de Ewald. Este valor é equivalente ao diâmetro da esfera de reflexões ou esfera de Ewald, construção geométrica que estabelece uma esfera de raio $\lambda^{-1}$ cujos pontos de rede recíproca devem atravessá-la para satisfazerem a Lei de Bragg e, portanto, ser observado o fenômeno da DRX. A esfera limite restringe o conjunto de pontos da rede recíproca que podem atravessar a esfera 
de Ewald. Portanto, todo conjunto de dados de DRX será sempre finito, e a equação da síntese de Fourier pode ser escrita para os valores limites dos índices $h k l$ como:

$$
\rho(x y z)=V^{-1} \sum_{h} \sum_{k} \sum_{l} F_{h k l} e^{-2 \pi i(h x+k y+l z)} .
$$

Experimentalmente, quando um monocristal de um dado composto é irradiado com raios $\mathrm{X}$, tipicamente com um 1 dentro da faixa compreendida entre $0,7 \AA$ a 2,0 $\AA$, ele se comporta como uma rede de difração tridimensional e produz um padrão de difração constituído de um arranjo tridimensional de intensidades difratadas. As intensidades destas reflexões estão intimamente relacionadas à densidade eletrônica dos átomos espalhadores, uma vez que o padrão de difração é a transformada de Fourier da densidade eletrônica da estrutura e, inversamente, a densidade eletrônica da estrutura é a transformada de Fourier do padrão de difração. Isto pode ser evidenciado através da relação anterior. Desta forma, através das intensidades e direções das reflexões difratadas é possível determinar a densidade eletrônica da estrutura.

Uma vez conhecidos os fatores de estrutura $F_{h k}$, a densidade eletrônica $\rho(x y z)$ pode ser calculada para cada ponto xyz na cela unitária, viabilizando a construção de mapas de densidade eletrônica de uma molécula em um cristal por um procedimento de cálculo conhecido como síntese de Fourier. Quando um mapa de densidade eletrônica com resolução em torno de 1,0 Å ao longo dos eixos cristalográficos é calculado, os picos de densidade eletrônica podem ser atribuídos aos centros espalhadores atômicos e, consequentemente, às suas posições. Todavia, o cálculo desses mapas envolve a obtenção das fases intrínsecas $\alpha_{h k l}$ dos fatores de estrutura, uma vez que:

$$
F_{h k l}=\mid F_{h k l} e^{i \alpha_{h k l}}
$$

\section{COLETA, PROCESSAMENTO E REDUÇÃO DOS DADOS}

A coleta de dados é o procedimento de medida das intensidades das ondas difratadas. Depois de um número convencional de reflexões, um computador acoplado ao difratômetro calcula os parâmetros da melhor cela unitária e da matriz de orientação, baseada em reflexões centradas e indexadas, seguindo a estratégia que dá um conjunto de dados completos e uma multiplicidade observada $(\mathrm{MoO})$ alta que é melhor conseguido com a tecnologia do detector bidimensional tipo Charge Coupled Device $\mathrm{CCD}^{8,10}$. A partir da matriz orientação, um programa de coleta de dados é iniciado de forma automática, sendo obtido um conjunto de imagens, em geral pelo método de rotação do cristal que, depois de integradas e processadas, levam a um conjunto de reflexões que fornecem tanto a direção $h k l$ quanto a intensidade de cada feixe difratado ${ }^{8}$. A partir do padrão de distribuição das intensidades obtidas, é possível se determinar os parâmetros de rede e a métrica que possibilitará a obtenção dos dados de simetria.

O próximo passo é a redução dos dados, uma vez que as intensidades das reflexões que foram medidas por difração de raios $\mathrm{X}$, estão afetadas por uma série de fatores que dependem do arranjo experimental utilizado. São obtidos, então, os módulos dos fatores de estrutura, $|F(\mathbf{h})|$, a partir das intensidades observadas experimentalmente, corrigidas pelos diversos fatores que os afetam que é executado por programas que aplicam várias correções como Lorentz, polarização, absorção, extinção primária e secundária, e determina os valores de "incerteza padrão" $(\sigma)$ para cada reflexão ${ }^{8,10}$.

Assim,os dados obtidos em um experimento de DRX são as intensidades espalhadas $I_{h k l}$ em direções definidas, as quais podem ser relacionadas apenas à amplitude dos fatores de estrutura e, portanto, aos seu módulos, através da relação:

$$
I_{h k l}=\frac{I_{\text {in }}}{\omega} K L_{h k l} p_{h k l} A_{h k l} e_{h k l}^{\prime}\left|\left(F_{h k l}\right)_{T}\right|_{\text {crist }}^{2},
$$

em que $I_{\text {in }}$ é a intensidade do feixe incidente de raios $X, \omega$ é a velocidade angular de rotação do cristal, $K$ é o fator de escala que converte os módulos dos fatores de estrutura em valores relativos para grandezas absolutas (definimos o fator de estrutura $F_{h k l}$ de um máximo de difração pela equação

$$
\frac{E_{\mathrm{esp}(h k l)}}{E_{\text {in }} f_{\mathrm{e}}},
$$

a qual nos permite concluir que tais fatores de estrutura $F_{h k l}$ são grandezas relativas, normalizadas em relação ao produto $E_{\text {in }} f_{\mathrm{e}}$, então, para levar $F_{h k l}$ para um valor absoluto devemos multiplicá-lo tanto por $E_{\text {in }}\left(I_{\text {in }}=E_{\text {in }}{ }^{2}\right)$ 
quanto por $f_{\mathrm{e}}$, termo este embutido no fator de escala $K$ ( $K=f_{\mathrm{e}}^{2} N^{2}$, onde $N$ é o número total de celas unitárias contidas dentro do volume $V_{\text {crist }}$ do cristal)), $L_{h k l}$ é o fator de Lorentz que corrige as diferentes oportunidades temporais e geométricas que diferentes pontos da rede recíproca têm para difratar em função da intersecção com a esfera de Ewald $\left(L_{h k l}=1 /\right.$ sen $\left.2 \theta\right), p_{h k l}$ é o fator de polarização discutido anteriormente, $A_{h k l}$ é o termo que corrige o efeito da absorção dos raios $\mathrm{X}$ e assume a forma de $e^{-\mu x}$, onde $\mu$ é o coeficiente de absorção linear do cristal e $x$ é trajetória total cursada pelos feixes incidente e espalhado, $e^{\text {' }}{ }_{h k l}$ corrige os efeitos de extinção primária (reflexões múltiplas por planos de Bragg fortemente espalhadores dos raios $\mathrm{X}$ ) e secundária (decréscimo da radiação incidente dentro do interior do cristal devido à difração por planos mais externos) e é função do número de planos perfeitamente paralelos dentro do cristal, entre outras variáveis. Por fim, o quadrado do módulo presente na equação que corrige as intensidades dos máximos de difração de raios $\mathrm{X}$ considera o fator de estrutura já corrigido em função da temperatura, em que:

$$
\left(F_{h k}\right)_{T}=\sum_{j}\left(f_{j}\right) T e^{2 \pi i\left(h x_{j}+k y_{j}+l_{z j}\right)} .
$$

$\mathrm{Na}$ prática, o processamento das intensidades experimentais de DRX é feito usando programas como o HKL Denzo-Scalepack ${ }^{11}$. Além dos fatores físicos e geométricos descritos anteriormente, este programa inclui outros parâmetros refináveis no cálculo do fator de escala geral das reflexões coletadas usando difratômetros com geometria Kappa ${ }^{12}$.

\section{SOLUÇÃO E REFINAMENTO DA ESTRUTURA}

A etapa de solução da estrutura está associada à obtenção da fase $\varphi(\mathbf{h})$ de cada reflexão medida, de forma que seja possível a construção dos mapas de densidade eletrônica $\rho(\mathbf{r})$. Ainda que não exista uma solução geral para o problema da fase, foram descobertas certas relações matemáticas entre fatores de estrutura a partir das quais certo conjunto de fases pode ser encontrado diretamente, os conhecidos Métodos Diretos ${ }^{3,8}$. O uso destas relações e outras levaram aos mais sofisticados códigos de resolução de estruturas, como sejam os desenvolvidos por George Sheldrick (SHELXS-97) ${ }^{13}$ e por Altomare e colaboradores (SIR-92) ${ }^{14}$ presentes no pacote de programas de resolução e análises cristalográficas WinGX ${ }^{15}$.

Além dos Métodos Diretos, que é amplamente utilizado na solução do problema da fase para pequenas moléculas sendo também um método promissor para a cristalografia de proteínas, existe outro método amplamente utilizado na determinação da posição de átomos pesados na cela unitária, conhecido como Método de Patterson ${ }^{16,17}$. Este método pode ser muito efetivo na localização do ponto de partida para a solução da estrutura, fornecendo informações sobre distâncias interatômicas a partir das quais é possível, eventualmente derivar as posições dos átomos sem que seja preciso informação acerca das fases. A solução de Patterson geralmente contém somente a sub-estrutura enquanto que os Métodos Diretos frequentemente dão as posições de quase todos os átomos não hidrogenóides, no entanto o Método de Parterson é mais rápido e os átomos remanescentes são encontrados geralmente na síntese Fourier diferença após alguns ciclos de refinamento ${ }^{3,6,16}$. Algumas estruturas que não podem ser resolvidas com os Métodos Diretos convencionais são resolvidas rapidamente com programas baseados nos algorítimos de espaço dual, como por exemplo, a implementação dos programas Shake and Bake ou SHELXD ${ }^{18}$. A escolha do melhor destes métodos vai depender do problema individual (tamanho da estrutura, presença de átomos pesados, presença de espalhamento anômalo, resolução máxima, e outros) ${ }^{10}$.

O que se obtém da resolução são os mapas de densidade eletrônica que são calculados utilizando o melhor conjunto de fases como indicados pelas figuras de mérito. Estes mapas resultam da síntese de Fourier usando como coeficientes da série, os fatores de estrutura normalizados $E(\mathbf{h})$. Os máximos num mapa de $E$ devem corresponder as posições atômicas, mas, por causa dos erros sistemáticos nas fases, podem existir picos residuais onde não existem átomos, ou faltar picos onde deveria ter devido a presença de algum átomo. O programa SHELXS- $97^{13}$ faz uma lista de picos, colocando-os em forma decrescente. Em seguida o operador deve tentar resolver a estrutura da molécula a partir deste mapa, através de critérios estéreo-químicos para identificação de fragmentos moleculares. Em alguns casos, boa parte das moléculas pode ser visualizada, porém, o mais comum é achar alguns picos e então usar, 
por exemplo, uma síntese Fourier Diferença que é uma síntese de Fourier na qual se utilizam como coeficientes a diferença entre os módulos dos fatores de estrutura observados $|F(\mathbf{h})|_{\text {obs }}$ e calculados $|F(\mathbf{h})|_{c a l}$ conforme a Eq. (32), sendo $\alpha_{\text {cal }}$ as fases calculadas a partir do modelo, e $k$ um fator de escala ${ }^{6,8}$.

$$
\Delta \rho(\mathbf{r})=\frac{1}{\mathrm{~V}} \sum_{\mathbf{h}}\left(k|\mathrm{~F}(\mathbf{h})|_{\text {obs }}-|\mathrm{F}(\mathbf{h})|_{\text {cal }}\right) \exp \left(\mathrm{i} \alpha_{\text {cal }}\right) \exp (-2 \pi \mathrm{ih.r}),
$$

este método é feita a diferença entre a densidade eletrônica observada $\rho(\mathbf{r})_{\text {obs }}$ e a densidade eletrônica calculada $\rho(\mathbf{r})_{\text {cal }}$, em que $\Delta \rho(\mathbf{r})=\rho(\mathbf{r})_{\text {obs }}-\rho(\mathbf{r})_{\text {cal }}$. A partir da Eq. (32) pode ser construído um mapa de densidade eletrônica do qual podem ser observados novos picos que podem ser atribuídos a átomos leves que não haviam sido incluídos no modelo inicial. Para estruturas contendo um ou mais átomos bem mais pesados em relação aos outros mais leves, os picos correspondentes a estes átomos mais leves ficam confundidos com as flutuações de fundo, aparecendo somente os picos correspondentes aos átomos pesados, conforme apresentado na Figura 1.

$\mathrm{Na}$ diferença das densidades eletrônicas estas flutuações anulam-se, pois são praticamente as mesmas em ambos e, portanto é possível localizar outros átomos. Em certos casos favoráveis é possível localizar, mediante esta síntese, a posição dos átomos de hidrogênio $0^{6,8}$. As posições atômicas da estrutura molecular inicial, obtidas da primeira solução, não são resultado direto do experimento de difração, mas uma interpretação da função densidade eletrônica calculada a partir da medida das intensidades e dos ângulos de fase de alguma forma determinados inicialmente. Novas fases $\varphi(\mathbf{h})$ mais acuradas podem ser calculadas para as posições atômicas, que permitem uma redeterminação da função de densidade eletrônica $\rho(\mathbf{r})$ com uma precisão maior. Novos átomos podem ser introduzidos e algumas vezes, precisam ser removidos do modelo para o melhor ajuste da função $\rho(\mathbf{r})$. Quando o modelo atômico está completo, os átomos podem ser descritos como elipsóides (refinamento anisotrópico) e as posições de átomos de hidrogênio podem ser determinadas ou calculadas geometricamente ${ }^{6,10}$.

Todas as etapas desde a obtenção do modelo inicial até a obtenção do modelo acurado é chamado de refinamento. Proporcionalmente as intensidades coletadas, se obtêm os fatores estrutura denominados observados $|F(\mathbf{h})|_{o b s}$. Baseado em transformadas de Fourier, um conjunto completo de fatores de estrutura denominados calculado $|F(\mathbf{h})|_{\text {cal }}$ é obtido a partir do modelo atômico. O refinamento é o procedimento de minimização da discordância entre os módulos dos fatores de estrutura observados $|F(\mathbf{h})|_{\text {obs }}$ e calculado $|F(\mathbf{h})|_{\text {cal }}$, realizando pequenas modificações nos parâmetros atômicos (portanto das fases $\varphi(\mathbf{h})$ calculadas) determinados para a estrutura aproximada ${ }^{3,6,19}$. O princípio utilizado é o dos Mínimos Quadrados ${ }^{10}$ em que a soma dos erros ao quadrado é minimizada através da função $M$ dada pela Eq. (33), onde $w$ é o peso atribuído a cada reflexão, inversamente proporcional ao desvio padrão $\sigma_{\mathbf{h}}$ que está associado à medida da reflexão $\mathbf{h}$. As intensidades calculadas são então comparadas com as intensidades medidas, e o melhor modelo é aquele que minimiza $M$.

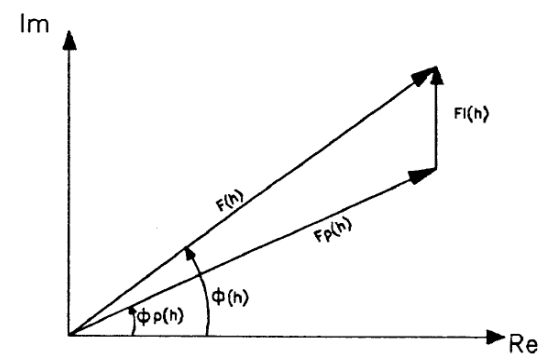

Figura 1. $\mathrm{O}$ fator estrutura $F(\mathrm{~h})$ de fase $\varphi(\mathrm{h})$ e suas componentes devido a átomos leves $F_{p}(\mathrm{~h})$ e pesados $F_{l}(\mathrm{~h})$.

$$
M=\sum_{\boldsymbol{h}} w_{\boldsymbol{h}}\left[|F(\boldsymbol{h})|_{\text {obs }}^{2}-|F(\boldsymbol{h})|_{\text {cal }}^{2}\right]^{2}
$$

O refinamento é um processo interativo na construção do modelo cristalográfico, guiado pela convergência e pela consistência química do modelo obtido ${ }^{2,10}$. Um dos programas mais utilizados para o refinamento de pequenas moléculas é o SHELXL ${ }^{13}$, cuja rotina está baseada no esquema da Figura 2.

Para utilizar o SHELXL somente dois arquivos de entrada (input) são necessários: o arquivo de instrução name.ins que contém os dados do cristal, as instruções de refinamento e as coordenadas dos átomos; e o arquivo com os dados de reflexão name.hkl que contém as coordenadas das reflexões medidas no espaço recíproco $h k l$ e as intensidades $F^{2}$ com suas incertezas associadas 
$\sigma\left(F^{2}\right)$. Todos os arquivos aqui citados estão no formato de arquivos de texto do tipo ASCII. Após alguns ciclos de refinamento com o SHELXL, são gerados dois arquivos principais. Um arquivo name.res com as novas coordenadas fracionárias e parâmetros de deslocamento atômicos obtidos, juntamente com os índices estatísticos utilizados na validação do modelo. O outro arquivo obtido é o name.lst que contém informações mais detalhadas do refinamento. Novos parâmetros podem ser incluídos ou ajustados no arquivo name.res, que poderá ser salvo na extensão name.ins, recomeçando todo o ciclo até a obtenção do modelo esperado ${ }^{10,13}$.

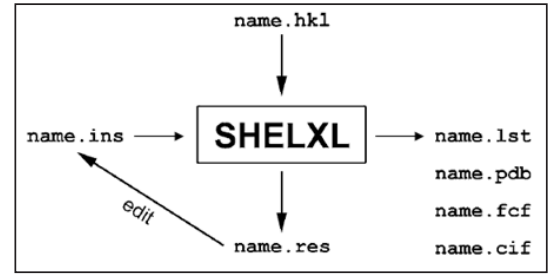

Figura 2. Organização da rotina de refinamento de estrutura utilizando o SHELXL.

Esperava-se que com a evolução da informática e dos equipamentos de coleta de dados fossem criadas estratégias automatizadas de refinamento de estruturas, mas um número crescente de estruturas tem requerido a intervenção e a percepção humana. Se o refinamento de uma estrutura não converge de um jeito normal, algumas possíveis ações podem ser executadas. Problemas durante o refinamento aparecem quando os fatores $\mathrm{R}$ crescem incontrolavelmente, e os parâmetros de deslocamento atômicos assumem valores absurdos, ou mudanças de parâmetros fazem a estrutura irreconhecível. Nos últimos estágios isto pode significar a extinção de alguns parâmetros ou ainda que algum parâmetro de deslocamento se torne significativamente negativo. Exclusão de átomos válidos é geralmente menos prejudicial do que a inclusão de falsos, e em qualquer caso, os átomos válidos reaparecem em mapas posteriores ${ }^{6,10,18}$.

A estrutura cristalográfica é a média espacial de todas as moléculas sobre o volume de um cristal em um determinado momento. Em muitos casos, partes da molécula são encontradas em mais de uma orientação cristalográfica. Essa situação pode ser ocasionada pela existência de mais de uma molécula na unidade assimétrica, a ocorrência de geminação ou ainda de desordem. Moléculas desordenadas possuem fragmentos que apresentam duas ou mais orientações, ou seja, dois ou mais conjuntos de coordenadas para cada átomo. Um indicativo de estrutura desordenada são valores muito altos os chamados parâmetros de deslocamento anisotrópico, $U_{\text {anis }}$. Os principais tipos de desordem são: substitucional e posicional, esta última podendo ser dinâmica ou estática. Na desordem substitucional, um mesmo sítio em duas celas unitárias, é ocupado por dois tipos diferentes de átomos. Na desordem posicional, um átomo ocupa dois ou mais sítios, podendo ser em uma mesma cela unitária (desordem dinâmica) ou em duas ou mais celas unitárias diferentes (desordem estática). Na desordem estática a média do conteúdo da cela unitária é obtida medindo todo o espaço sobre diferentes celas unitárias. A desordem dinâmica pode ser distinguida da desordem estática realizando a coleta de dados em duas temperaturas distintas, visto que a vibração térmica diminui quando a temperatura se reduz. Um caso especial de desordem, chamado mess, ocorre quando grandes vazios ou cavidades no interstício molecular são preenchidos por moléculas do solvente, orientadas de maneira randômica, que não contribuem no padrão de difração já que seu espalhamento é difuso. A introdução de desordem no modelo aumenta consideravelmente o número de parâmetros a serem refinados. Portanto a fim de melhorar o refinamento pode-se utilizar restraints e constraints co,10,18. $^{3,}$

Constraints são equações que relacionam ou atribuem valores numéricos fixos a dois ou mais parâmetros reduzindo o número de parâmetros independentes a serem refinados. Restraints são ferramentas que permitem ao cristalógrafo introduzir informações químicas e físicas provenientes de outras fontes que não o experimento de difração de raios $\mathrm{X}$, no processo de refinamento. Os restraints são utilizados a fim de assegurar que certos parâmetros geométricos e de deslocamento térmico estejam dentro da normalidade, restringindo distâncias, ângulos de ligação e ângulos torsionais a certos valores. Estruturas desordenadas são freqüentemente tratadas e modeladas utilizando os softwares SHELXL-97 ${ }^{13}$ e PLATON $^{20}$, ambos integrantes do pacote WinGX ${ }^{15}$. 


\section{VALIDAÇÃO, ANÁLISE DO MODELO E DEPÓSITO NO BANCO DE DADOS CRISTALOGRÁFICO}

Por ser a cristalografia uma metodologia estrutural, ocorre durante as etapas de determinação estrutural, uma série de erros que interferem na validade do resultado obtido. A análise destes possíveis erros é feita durante a etapa de validação que irá avaliar a exatidão e a precisão desses resultados sendo de grande importância para a qualidade e confiabilidade das estruturas resolvidas. Outra preocupação está associada à qualidade da análise, e depende, entre outros fatores, da qualidade do cristal, da coleta de dados, da experiência do cristalógrafo e dos programas utilizados. Os critérios analisados são: coerência química na estrutura, o conjunto dos dados, a precisão nas distancias e ângulos de ligação, a qualidade dos índices estatísticos, a vibração térmica dos átomos, as interações intermoleculares, a desordem no cristal e a completeza do espaço tridimensional cristalino. Índices estatísticos são utilizados para avaliar a diferença entre o conjunto de dados teóricos $\Sigma|F(\mathbf{h})|_{\text {cal }}$, gerado para o modelo construído, e o conjunto de dados experimentais $\Sigma|F(\mathbf{h})|_{o b s}$ medido a partir do cristal que contém o erro randômico ${ }^{2,3}$.

Uma avaliação estatística fazendo uso dos conjuntos $\Sigma|F(\mathbf{h})|_{o b s}$ e $\Sigma|F(\mathbf{h})|_{c a l}$ pode ser feita com Figuras de Mérito, que são índices estatísticos que indicam a qualidade de uma estrutura cristalográfica, sempre obtidos pela comparação entre os fatores de estrutura medidos experimentalmente e aqueles calculados teoricamente. $\mathrm{O}$ grau com que a distribuição das diferenças entre os conjuntos $\Sigma|F(\mathbf{h})|_{o b s} \mathrm{e}$ $\Sigma|F(\mathbf{h})|_{\text {cal }}$ se ajusta aos valores esperados, afetados pelos erros randômicos, pode ser avaliado através do teste do $X^{2}$ (chi-quadrado) ${ }^{3,21}$, dado pela expressão do Goodness of Fit (Goof) ou simplesmente $S$ (para o SHELXL-97),

$$
\text { Goof }=\left[\sum w\left(|F(\mathbf{h})|_{\text {obs }}^{2}-|F(\mathbf{h})|_{c a l}^{2}\right)^{2} / m-n\right]^{1 / 2},
$$

onde $m$ corresponde ao número de observações e $n$ ao número de parâmetros independentes refinados ( $G$ ou fator de escala, posições atômicas, parâmetros de ocupação e outros) utilizados no refinamento pelo métodos dos Mínimos Quadrados. Goof > 5 indica algum problema com um dos conjuntos $\Sigma|F(\mathbf{h})|_{o b s}$ ou $\Sigma|F(\mathbf{h})|_{c a l} \mathrm{e}$ Goof, significativamente menor do que a unidade sugere que o modelo é melhor do que os dados e, portanto, não tem significado físico. A unidade é seu valor idea ${ }^{10}$. O grau de precisão do modelo cristalográfico construído e a estrutura real pode ser avaliado pelos índices de discordância (residual factors), calculado a partir do quantitativo total desviado entre os conjuntos $\Sigma|F(\mathbf{h})|_{\text {obs }} \mathrm{e}$ $\Sigma|F(\mathbf{h})|_{\text {cal }}$, dado pela Eq. (35).

$$
R=\left.\sum|| F(\mathbf{h})\right|_{o b s}-\left.|F(\mathbf{h})|_{c a l}\left|/ \sum\right| F(\mathbf{h})\right|_{o b s} .
$$

Para uma estrutura confiável, o valor de $R$ deve estar entre 0,02 e 0,06 após o refinamento. Elevados ao quadrado todos os fatores de estrutura associados, os correspondentes às reflexões mais intensas têm um peso maior em relação aos correspondentes às reflexões fracas, sendo obtida assim a Eq. (36) com os índices de discordância ponderados $R_{w}$ ou $w R_{2}$.

$$
R_{w}=\left[\sum w\left(|F(\mathbf{h})|_{o b s}^{2}-|F(\mathbf{h})|_{c a l}^{2}\right)^{2} / \sum w\left(|F(\mathbf{h})|_{o b s}^{2}\right)^{2}\right]^{1 / 2} .
$$

Finalizado o trabalho de construção do modelo estrutural, todas as informações cristalográficas podem ser armazenadas num arquivo texto comum, respeitando o padrão Crystallographic Information File (CIF), adotado em 1991 pela International Union of crystallography (IUCr). Esse formato de arquivo armazena cada informação cristalográfica através de um código para o item no formato ASCII. O senso químico de uma estrutura cristalina deve ser prioritário sobre todos os métodos estatísticos ${ }^{2,10,18}$.

Obtido o modelo cristalográfico validado e com sentido químico, são realizadas análises da geometria molecular (distâncias e ângulos entre átomos, e ângulos diedrais), além de estudos supramoleculares analisando como as moléculas se organizam no cristal (estrutura cristalina), em decorrência das interações intermoleculares. Estas interações são mais fracas do que as ligações intramoleculares (interações entre átomos em uma mesma molécula que podem ser iônica, covalente ou metálica). As interações intermoleculares incluem interações eletrostáticas fortes (entre íons ou entre íons dipolo), forças de Van der Waals e dentre estas as forças de dispersão (forças de London), ligações de hidrogênio, interações hidrofóbicas e outras interações envolvendo sistemas $\pi$ deslocalizados ou anéis aromáticos ${ }^{9,22}$. 
Com exceção das interações eletrostáticas fortes, as ligações de hidrogênio são as interações de maior energia (4$60 \mathrm{kj} / \mathrm{mol}$ ) em cristais moleculares afetando a maneira com que as moléculas são empacotadas ${ }^{9}$. A ligação de hidrogênio é por si só a mais relevante interação intermolecular na natureza, sendo o principal fator na determinação da estrutura da água, enovelamento de proteínas e emparelhamento das bases no DNA. Esta interação consiste em um átomo de hidrogênio que fica entre dois átomos eletronegativos, e um par de elétrons isolados, especificamente nitrogênio $(N)$, oxigênio $(O)$ ou halogênios $(F, C l, B r)$. As ligações de hidrogênio são altamente direcionais e a energia eletrostática geralmente é o termo de maior contribuição para a sua estabilidade. As ligações de hidrogênio chamadas clássicas incluem os sistemas $O-H \ldots O, N-H \ldots O$ e $O-H . . . N$. As chamadas não clássicas são: $C-H . . . O, O-H \ldots \pi$, e $C-H \ldots \pi$. Enquanto que as liagações de hidrogênio $O-H . . . O, N-H . . . O$ vem sendo estudadas por décadas, o mesmo não pode ser dito para as interações não clássicas como $C-H . . . O, O-H \ldots \pi$, que somente a algum tempo vem ganhando aceitação de ser uma genuína ligação de hidrogênio ${ }^{23,24}$. As ligações $C-H$...O fazem contribuições energeticamente favoráveis para a estabilidade do empacotamento das moléculas.

Para a identificação destas interações é necessário a utilização de critérios tais como distância doador $D$ / aceptor $A$ e o ângulo $D-H . . . A$. A distância $D$...A é um dos atributos importantes para determinar a existência deste tipo de ligação e deve ser menor que a soma dos raios de Van der Walls do átomo doador e do átomo aceptor. Assim, para uma ligação de hidrogênio entre átomos de carbono e oxigênio, se o raio de Van der Walls do átomo de oxigênio é 1,40 Å e do átomo de carbono ligado ao hidrogênio é de $1,84 \AA$, então a distância C...O deve ser menor que $3,25 \AA$ para ter uma interação deste tipo. A distância D/A é uma condição necessária, mas não suficiente, pois como mencionado estas interações são direcionais de forma que o ângulo ao redor do átomo de hidrogênio deve ser o mais linear possível ${ }^{9}$. Nestas interações a variação do ângulo aceita está entre $100^{\circ}$ e $180^{\circ}$.

As ligações de hidrogênio são classificadas de acordo com sua topografia como intramolecular, intermolecular ou bifurcada, e de acordo com sua energia, como fraca, moderada e forte. Além das ligações de hidrogênio intramoleculares, embora menos comum e muitas vezes mais fraca do que as ligações de hidrogênio intermoleculares, também podem exercer um impacto considerável sobre as propriedades moleculares. Elas incluem a estabilidade relativa dos isômeros estruturais, a distribuição de cargas nas moléculas, a reatividade e outros. Em uma típica ligação de hidrogênio $D-H \cdots A$ o átomo de hidrogênio é ligado covalentemente a um átomo eletronegativo $D$, e não-covalentemente a um átomo eletronegativo $A$. Por causa de sua longa série de interações, as ligações de hidrogênio também são encontradas entre um doador e dois aceptores, ou entre um aceptor e dois doadores. O primeiro tipo é normalmente conhecido como bifurcada ${ }^{9,24}$.

As interações $C$ - $H \ldots \pi$ são outro tipo de ligações de hidrogênio não clássicas que são frequentemente encontradas no arranjo supramolecular de proteínas e pequenas moléculas orgânicas contendo elétrons $\pi$ deslocalizados ou anéis aromáticos. Em uma análise das interações $C-H . . . \pi$ em estruturas depositadas no Protein Data Bank (PDB) foi observado que a maioria delas apresentava uma distância $d$ (mostrada na Figura 3A) de 3,6 $\AA$ entre o átomo doador e o centro de massa da ligação $\pi$ ou centro de massa de um anel aromático $C_{g}$ (centróide) sendo observado ainda que para incluir $96 \%$ de todos os dados encontrados essa distância deveria ser ampliada a 4,5 $\AA^{23,24}$. Como no caso das outras interações envolvendo átomos de hidrogênio, o ângulo $C-H . . . C_{g}$ é importante e o valor mínimo aceito é $120^{\circ}$.

Ultimamente, vem ganhando atenção e destaque outro tipo interação que ocorre em sistemas aromáticos: o empilhamento $\pi$ - $\pi$ também conhecido como $\pi$-stacking (Figura 3B e 3C). Por definição, as interações $\pi-\pi$ são contatos hidrofóbicos que ocorrem entre dois sistemas $\pi$ deslocalizados. De acordo com estudos sobre a natureza das interações $\pi$ - $\pi$, ressalta-se que a energia de interação entre as moléculas resulta do somatório de várias energias que podem ser observadas na Eq. (37) ${ }^{25}$

$$
E_{\text {Total }}=E_{\text {eletrostático }}+E_{\text {indução }}+E_{\text {dispersão }}+E_{\text {repulsão }} \text {. }
$$

A maior contribuição sobre a geometria dessas interações é devido aos efeitos eletrostáticos, decorrentes do quanto deslocado estará o sistema de anéis e quais são os anéis interagentes ${ }^{25}$. Como pode ser observado na equação, também existem outros fatores que contribuem energeticamente. Ressalta-se que pelas informações 
cristalográficas não se pode ter acesso a essas energias, mas a distância que separa as moléculas pode ser um parâmetro que forneça uma idéia do quão forte é a interação.

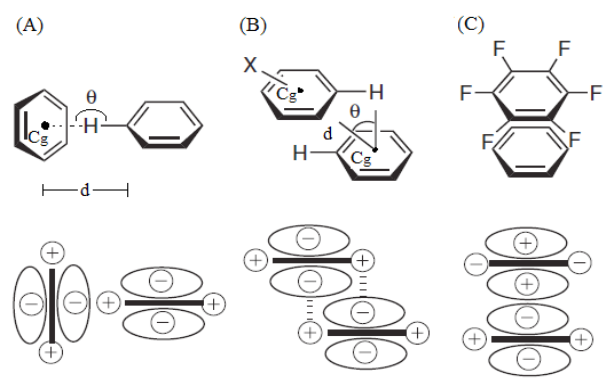

Figura 3. Geometria das interações entre anéis aromáticos. (A) interação vértice-face ou forma T. (B) Empilhamento face a face deslocada. (C) Empilhamento face a face.

Uma das distâncias que podem ser utilizadas para identificar uma interação $\pi$ - $\pi$ é à distância interplanar, ou seja, a distância entre dois planos que são formados por sistemas aromáticos. Como pode ser observado na 4B, as moléculas não se arranjam de maneira alinhada uma sobre a outra, pois isto resultaria em uma repulsão entre elétrons $\pi$. Assim as moléculas estão ligeiramente deslocadas, sendo que este deslocamento apresenta um ângulo aproximado de $20^{\circ}$. Com isto, outro parâmetro é a distância entre centróides $C_{g}$, é comumente utilizado para estudar as interações $\pi-\pi$. O fato deste não ser um contato perpendicular implica que sua distância sempre seja maior do que a encontrada por um contato interplanar'. Geralmente as distâncias $C_{g} \ldots C_{g}$ apresentam um intervalo de 3,4 a $3,8 \AA$. Porém esta faixa de distâncias intecentróides pode assumir limiter inferiores e superiores diferentes destes valores ${ }^{9,25}$.

Após toda a etapa de validação e análise de dados, o modelo cristalográfico no formato CIF, pode ser depositado no banco de dados apropriado. Para pequenas moléculas geralmente se utiliza o Cambridge Structural Database CSD mantido pelo Cambridge Crystallographic Data Centre (CCDC). O Protein Data Bank (PDB) é utilizado para proteínas e macromoléculas biológicas.

\section{Considerações finais}

A cristalografia por difração de raios X apresentase como uma ferramenta de alta aplicabilidade no trabalho de caracterização de compostos cristalinos, permitindo o entendimento da relação entre a estrutura e as propriedades físico-químicos dos compostos. Através dessa metodologia podem-se determinar as posições relativas de todos os átomos que constituem a molécula (estrutura molecular) e a posição relativa de todas as moléculas que constituem a cela unitária e o cristal (estrutura cristalina). Pode-se, portanto, estabelecer os ângulos e as distâncias interatômicas da estrutura cristalina, bem como as interações intermoleculares (Van der Waals, dipolo-dipolo, ligação de hidrogênio, entre outras interações não-covalentes). Em casos complexos, assim como estruturas com desordem, pseudo-simetria ou geminação, o conhecimento cristalográfico, habilidades de refinamento e experiência são de vital importância para obter estruturas cristalinas de alta qualidade.

\section{Agradecimentos}

Os autores agradecem à CAPES pelo apoio financeiro.

\section{Referências}

1. Jensen, W. P.; Palenik, G. J.; Suh, I. H.; “The history of molecular structure determination viewed through the nobel prizes". J. Chem. Edu., 2003, v. 80, p. 753.

2. Napolitano, H. B.; Camargo, A. J.; Vencato, I.; Lariucci, C.; Ellena, J.; Cunha, S.; “Caracterização de Fármacos Polimórficos”. Revista Estudos, 2005, v. 32, p. 2021-2046.

3. Giacovazzo, C; Monaco, H. L.; Viterbo, D.; Scordari, F.; Gilli, G.; Zanotti, G.; Catti, M.; "Fundamentals of Crystallography". ed. Giacovazzo, Oxford: IUCR - Oxford University Press, $2^{a}$ ed., 2002.

4. Hammond, C.; "The Basics of Crystallography and Diffraction". Oxford: IUCr - Oxford University Press, $3^{\mathrm{a}}$ ed., 2009.

5. Drenth, J.; “Principles of Proteins X-Ray Crystallography”. New York: Springer Verlag, 1994.

6. Clegg, W.; “Crystal Structure Analysis: Principle and Pratices”. New York: IUCr - Oxford University Press, $2^{\mathrm{a}}$ ed., 2009.

7. Glusker, J. P.; Lewis, M.; RossI, M.; “Crystal Structure Analysis for Chemists and Biologists”. Oxford: VCH, 2a ed., 1994.

8. Stout, G. H.; Jensen, L. H.; "X-Ray Structure Determination”. New York: Wiley-Interscience, $2^{\mathrm{a}}$ ed., 1989.

9. Steed, J. W.; Atwood, L. J.; "Supramolecular Chemistry". Weinhein: Wiley-VHC, 2009.

10. Müller, P.; Irmer, R. H.; Spek, A. L.; Schneider, T. R.; Sawaya, M. R.; "Crystal Structure Refinement: A Crystallographer's Guide to SHELXL” New York: IUCr - Oxford University Press, 2006. 
11. Otwinowski, Z.; Minor, W. "Processing of X-ray diffraction data collected in oscillation mode.” In: Carter Junior, C. W.; Sweet, R. M. (Eds.). "Methods in Enzymology: macromolecular crystallography”. New York: Academic Press, 1997. v. 276, parte A. p. 307-326.

12. Otwinowski, Z.; Borek, D.; Majewski, W.; Minor, W. "Multiparametric scaling of diffraction intensities." Acta Crystallogr. Sect. A, 2003, v. 59, p. 228-234.

13. Sheldrick, G. M.; "A short history of SHELX”. Acta Crystallographica Section A, 2008, v. 64, p. 112-122.

14. Altomare A. A.; Cascarano, G.; Giacovazzo, C.; Guagliardi, A.; "SIR92 - A program for crystal structure solution". J. Appl. Cryst., 1993, v. 26, p. 343-350.

15. Farrugia, L. J.; "WinGX - suite for small molecule single crystal crystallography”. J. Appl. Cryst., 1999, v. 32, p. 837-838.

16. Shmueli, U.; "Theories and Techniques of Crystal Structure Determination”. New York: Oxford University Press - IUCr, 2007.

17. Usón, I.; Sheldrick, G. M.; “Advances in direct methods for protein crystallography”. Biophysical Methods, 1999, v. 9, p. 643-648.

18. Müller, P.; "Practical suggestions for better crystal structures". Crystallography Reviews, 2009, v. 15, p. 57-83.

19. Sheldrick, G.M. “The SHELX-97 Tutorial”. 1997.

20. Spek, A. L.; "Platon / Pluton”. Acta Cryst. A, 1990, v. 46.

21. Schwarzenbach, D. “Crystallography”. Chichester: J. Wiley e Sons, 1996.

22. LI, W.; Cheng, J.; Gong, B.; Sun, J.; "Effect of methyl group on the cooperativity between cation- $\pi$ interaction and $\mathrm{NH}$...O hydrogen bonding”. Journal of Molecular Structure, 2008, v. 867, p. 107-110.

23. Brandl, M.; Weiss, M. S.; Jabs, A.; Sühnel, J.; Higenfeld, R.; “C-H... $\pi$ in proteins". J. Mol Biol., 2001, 307, p. 357.

24. Desiraju, G. R.; "The C-H...O hydrogen bond in crystals: what is it?”. Acc. Chemical Res., 1991, v. 24, p. 290.

25. Hunter, C. A.; Sanders, J. K. M.; "The nature of $\pi-\pi$ interactions". Journal of Americal Chemical Society, 1990, n. 14, v. 112, p. 5525-5534.

\section{William B. Fernandes ${ }^{1 *}$, Hamilton B. Napolitano ${ }^{1}$, Caridad Noda- Perez $^{2}$, Felipe T. Martins ${ }^{2} \&$ Carlito Lariucci $^{3}$.}

${ }^{1}$ Ciências Exatas e Tecnológicas, UEG, Caixa Postal 459, CEP 75001970, Anápolis, GO.

${ }^{2}$ Instituto de Química, UFG, Caixa Postal 131, CEP 74001-970, Goiânia, GO.

3nstitutode Física, UFG, Caixa Postal 131, CEP74001-970, Goiânia, GO.

*e-mail: william.fernandes@ueg.br 